\title{
Fine-Needle Aspiration Biopsy of Liver Lesions Yields Higher Tumor Fraction for Molecular Studies: A Direct Comparison With Concurrent Core Needle Biopsy
}

\author{
Patricia Ellen Goldhoff, MDa,*; Poonam Vohra, MDª,; Kanti Pallav Kolli, MDc; and Britt-Marie Ljung, MDa
}

\section{ABSTRACT}

Background: This retrospective study evaluated and compared the diagnostic accuracy and suitability of tissue specimens for advanced molecular diagnostic testing obtained via 2 different techniques for percutaneous biopsy of primary and metastatic liver tumors. Patients and Methods: Samples from 137 patients with liver masses who underwent concurrent fine-needle aspiration biopsy with cell block (FNAB-CB) and core needle biopsy (CNB) at 2 hospitals were assessed for diagnostic accuracy, tumor fraction, and tumor cellularity. A subset of FNAB-CBs, that were deemed to have less or equal tumor cellularity compared with CNBs, had level sections performed and were reassessed for tumor cellularity. Results: Diagnostic accuracy was $96 \%$ for FNAB and $93 \%$ for CNB $(P=.267)$. In FNAB-CBs, tumor fraction was significantly higher than in CNB samples (67\% vs $36 \% ; P<.0001)$, whereas nontumor components were significantly lower (stromal component, $7 \%$ vs $29 \% ; P<.0001$; background benign hepatocytes, $25 \%$ vs $36 \% ; P=.003$ ). Additionally, in $44 \%$ of cases, FNAB-CB tumor cellularity was equal to or greater than that of the concurrent CNB. Conclusions: In the current age of personalized medicine, a minimally invasive, safe approach to obtaining adequate tissue for myriad molecular testing is paramount. We have shown that FNAB sampling is diagnostically accurate and produces higher tumor fractions than CNB. Thus, FNAB should be strongly considered as an initial sampling modality, especially for patients in whom molecular tests will determine management.

J Natl Compr Canc Netw 2019;17(9):1075-1081 doi: $10.6004 /$ jnccn.2019.7300

${ }^{a}$ Department of Pathology, University of California San Francisco; ${ }^{b}$ Department of Pathology, Zuckerberg San Francisco General Hospital; and 'Department of Radiology, University of California San Francisco, San Francisco, California.

${ }^{\star}$ Co-first authors.

\section{Background}

Image-guided percutaneous biopsy is one of the most common procedures performed in radiology departments, ${ }^{1}$ and the volume of these procedures is increasing. ${ }^{2}$ At the same time, rapid advances in both molecular diagnostic testing of tissue samples and the development of targeted therapeutics has led to the era of personalized medicine in oncology. The historical purpose of percutaneous biopsy to determine tumor histologic type and grade has been expanded to include determination of the molecular profile, which provides prognostic information and informs therapeutic decision-making. ${ }^{3}$

Differences in needle gauge and the associated expectation of lower tissue yield have led many to consider samples from fine-needle aspiration biopsy (FNAB) inferior to those from core needle biopsy (CNB). Conflicting data in the literature have also led to confusion. A recent study of 74 paired FNABs and CNBs of liver masses showed FNAB to have superior diagnostic accuracy in metastatic lesions, but CNB resulted in a slightly higher diagnostic rate for hepatocellular carcinoma. ${ }^{4}$ However, not all cases were sampled using both modalities, and no information was available on how many of the FNAB cases included a cell block, which is needed for common ancillary testing. In addition, numerous studies have shown FNAB to be a highly accurate, safe, and cost-effective tissue sampling method, ${ }^{5-8}$ and samples have been shown to be suitable for molecular testing, including next-generation sequencing (NGS) ${ }^{9-11}$ Therefore, as oncology moves into the era of personalized medicine, it is imperative to determine the utility and effectiveness of tissue sampling modalities.

The choice of sampling method should reflect the safest and most effective approach that will yield sufficient, high-quality tissue to allow both an accurate diagnosis and any additional studies that may affect prognosis and therapeutic options. Several factors drive the choice of sampling modality in current practice, including radiologist or ordering provider preference, available resources and skill sets, and research protocol criteria. At University of California San Francisco (UCSF), 
the cytology service is routinely involved in rapid onsite evaluation (ROSE) and specimen triaging at the time of sampling, thus contributing to a robust, diagnostic FNAB specimen. We have observed that FNAB sampling concentrates tumor cells while leaving most or all of the stromal and other nontumor components behind. The mechanics of FNAB sampling with a beveled needle favor the collection of typically loosely cohesive tumor cells over dense connective tissue, such as the tumorassociated stroma. Furthermore, FNAB allows precise targeting of the tumor area regardless of size and shape. However, CNBs sample all of the material in the target area, resulting in variable amounts of stroma that, depending on the composition of the tumor, often markedly dilute the tumor cells. In addition, CNB sampling, particularly of small targets, often includes adjacent benign components from tissue bordering the target lesion, resulting in a large proportion of CNB specimens deemed inadequate for molecular testing, diminished analytic sensitivity in specimens with borderline tumor concentration, and delayed treatment when repeat biopsy becomes necessary. ${ }^{12,13}$

Because formalin-fixed, paraffin-embedded (FFPE) tissue is the primary substrate for most currently available molecular tests, we reviewed concurrently acquired FNABs with cell block -cell blocks (FNAB-CBs) and CNBs of primary and metastatic liver lesions, and compared these sampling modalities in terms of overall diagnostic accuracy and tumor concentration and cellularity in the FNAB-CB component.

\section{Patients and Methods}

The anatomic pathology databases of 2 hospitals, UCSF Medical Center (UCSFMC) and Zuckerberg San Francisco General Hospital (ZSFGH), were queried for all CTor ultrasound-guided liver FNABs and CNBs performed from 1997 through 2016. Only concurrent CNBs and FNAB-CBs were included. In total, 137 consecutive cases meeting the above criteria were included. Diagnoses encompassed both primary and metastatic liver lesions and included the following in order of decreasing frequency: adenocarcinoma, hepatocellular carcinoma, neuroendocrine tumor, neuroendocrine carcinoma, cholangiocarcinoma, malignant neoplasm (not otherwise specified), melanoma, nasopharyngeal carcinoma, squamous cell carcinoma, gastrointestinal stromal tumor, lymphoma, and hepatic adenoma. This study was approved by the Institutional Review Boards of both hospitals. Tissue requirements for clinical trial enrollment vary, and whether tissue available in a given case will qualify depends on requirements for the individual clinical trial; in a subset of cases, additional tissue may need to be collected. In our institution (UCSF), cases in the study cohort were not included in enrollment in a clinical trial.
Interventional radiologists used ultrasound or CT guidance, typically with a coaxial introducer, to sample the liver lesion of interest. FNABs were performed using 22-gauge needles followed by CNB sampling obtained during the same visit using 18- to 20-gauge spring-loaded core biopsy needles. In all cases, direct smears were prepared from the FNAB material and stained with toluidine blue, and an intraprocedural ROSE was performed by a cytopathologist or cytopathology fellow. At both hospitals, CBs were attempted for all cases. Depending on the immediate assessment, an additional 1 to 2 FNAB passes or residual washout after expulsion of material for smears (fixed in $10 \%$ neutral buffered formalin) were dedicated to $\mathrm{CB}$ preparation even for cases in which CNB was obtained. In addition, material was deposited in RPMI media for flow cytometry if lymphoma was included in the differential diagnosis based on immediate evaluation. We do not have consistent documentation of the number of passes for FNA and the number of cores submitted for each case.

At one hospital, the $\mathrm{CB}$ was prepared using the collodion bag method, ${ }^{14}$ in which a conical glass test tube is filled with a commercially available liquid polymer, collodion (Cardinal Health Inc.). The collodion is then poured out of the test tube, leaving a lining along the walls of the tube, which is then inverted and allowed to dry completely (10-15 minutes) under a laminar flow hood. This process forms a membrane bag lining the inside of the tube. Preparation of collodion- lined tubes can be performed in batches, and the tubes can be stored filled with distilled water and covered for up to 2 weeks. When 20 tubes per batch are prepared, each tube takes approximately 2 minutes. The remainder of the CB preparation is similar in complexity to other commonly used techniques. The FNAB needle is rinsed into a $10-\mathrm{mL}$ vial of formalin, the entire sample is poured into the collodion-lined test tube, and the tube is centrifuged at $2,500 \mathrm{rpm}$ for 10 minutes to form a pellet. The membrane bag is then gently separated from the test tube wall, which forms a collodion membrane bag with the pellet at the bottom of the test tube. The bag is gently removed from the test tube. A cotton string is tied around the bag just above the cell pellet at the base and the bag is then placed sideways on the bench and cut just above the string. The bag containing the pellet is placed in a tissue cassette, embedded sideways, and processed as per routine histology. The other hospital used the agar CB method, wherein core biopsies were fixed in $10 \%$ buffered formalin, paraffin-embedded, sectioned, and stained with hematoxylin-eosin for microscopic evaluation.

FNAB and CNB specimens were assessed for the presence of diagnostic tissue. Tumor fraction and relative tumor cellularity of FNAB-CBs and CNBs were evaluated by a cytopathologist (P.E. Goldhoff) using light 
microscopy. Tumor fraction was defined as the percentage of nucleated tumor cells present and was averaged over the entire slide to include both tumor-rich and tumorpoor areas. Nontumor cell fractions, including tumor stroma and benign hepatocytes, were also estimated. Tumor cellularity was defined as the number of tumor cells present in a single tissue section and was compared between paired FNAB-CBs and CNBs. One of the sampling modalities was assigned as having more tumor cellularity or the samples were designated as containing an equal amount of tumor cells. Difficult or borderline cases were assessed by a second cytopathologist (B.M. Ljung).

Given the centrifugation and embedding process involved in preparing FNAB-CBs, cell distribution is not always uniform throughout the paraffin block, with initial sections often showing less material than what is present deeper in the block. Thus, a subset of cases with FNAB-CBs available and deemed to have less or equal tumor cellularity compared with CNBs were further analyzed $(n=81)$. A total of 19 level sections were prepared, and levels 1, 7, 13, and 19 were stained with hematoxylineosin and reviewed by a cytopathologist (P.E. Goldhoff). The levels were assessed for tumor cellularity, and a single section from a given FNAB-CB with the highest tumor cellularity was then compared with the paired CNB sample. CNB samples were routinely sectioned well into the core to provide optimal visualization of material representing the sample as a whole, thus making level sections highly unlikely to change the composition of the sample or to increase overall cellularity significantly.

We also retrieved blocks to attempt deeper levels in the small subset of CNB cases that showed minimal or no tumor initially $(n=12)$. In 8 of these cases, deeper levels were not obtained because 3 blocks were already exhausted and 5 cases were recent with potentially ongoing care, and it was determined that cutting through the block could interfere with medical care. The remaining 4 blocks were subjected to deeper levels because either they were from deceased patients or there was no evidence of ongoing medical care. Among these cases, 3 showed only liver tissue and no tumor and 1 showed almost all liver with a submillimeter focus of tumor at one end of a single core compared with abundant tumor cells present in the corresponding FNAB-CB.

Statistical analysis was performed with GraphPad Prism 6 (GraphPad Software, Inc.). Two group comparisons were conducted using either the McNemar's test for binary variables or the paired 2-tailed $t$-test in the case of continuous variables; $P<.05$ was considered significant.

\section{Results}

There was no significant difference in obtaining diagnostic tissue with an FNAB specimen versus a CNB specimen (Table 1). A diagnosis was rendered and recorded in the original reports issued to the patient's chart for 132 FNAB specimens (96\%) and $127 \mathrm{CNB}$ specimens (93\%) $(P=.267)$. For patient care purposes, $\mathrm{CNB}$ and FNAB specimens were evaluated independently with a separate diagnosis assigned to each. Both specimens were evaluated by a single pathologist at ZSFGH and by 2 separate pathologists at UCSFMC, each examining either the FNAB or the CNB. Microscopic review of the samples did not result in revision of any diagnosis.

The tumor concentration overall was significantly higher in FNAB-CB specimens than in CNB specimens (67\% vs $36 \%$, respectively; $P<.0001$ ) (Figure 1). Furthermore, analysis based on diagnosis/tumor type showed that FNAB-CBs had superior tumor concentration compared with CNBs in 14 of 17 categories, representing 130 of the 137 cases analyzed (Table 2). In all cases in which immunohistochemistry was indicated, the CBs accommodated the need. Based on whole sections, 25\% of FNAB-CBs and $43 \%$ of CNBs did not meet the cutoff of $20 \%$ tumor cells typically required for molecular testing. Nontumor components were also analyzed. Stromal components were significantly fewer in FNAB-CBs compared with CNBs $(7 \%$ vs $29 \%$; $P<.0001$; Figure 2). Similarly, fewer background benign hepatocytes (25\% vs $36 \%$; $P=.003$ ) were present in FNABCBs compared with CNBs (Figure 3). In addition to tumor concentration, overall tumor cellularity was compared. Two cases without tumor on the FNAB-CBs and CNBs, respectively, were excluded from the analysis. At initial review, FNAB-CBs exhibited more tumor cells in 35 cases (26\%), CNBs were more cellular in $81(60 \%)$, and equal amounts of tumor cells were noted in 19 (14\%).

Due to the processing of FNAB-CBs, it is not uncommon for cell distribution to vary within the paraffin blocks. Therefore, in cases for which FNAB-CB cellularity was equal to or less than that of $\mathrm{CNB}(n=81)$, level sections were obtained and cellularity was reevaluated and compared with the concurrent CNB. After level sections were reviewed, $20 \%$ of these FNAB-CBs (16/81)

Table 1. Diagnostic Rates Based on Biopsy Specimens ( $=137$ )

\begin{tabular}{|c|c|c|c|c|}
\hline & \multirow[b]{2}{*}{ Core Needle Biopsy } & \multirow{2}{*}{$\begin{array}{c}\text { Fine-Needle } \\
\text { Aspiration Biopsy }\end{array}$} & \multicolumn{2}{|c|}{ Cell Block Method } \\
\hline & & & Collodion Bag $(n=60)$ & Agar $(n=77)$ \\
\hline Diagnostic rate, \% (n) & $93(127)$ & $96(132)$ & $81.6(49)$ & $81.8(63)$ \\
\hline
\end{tabular}




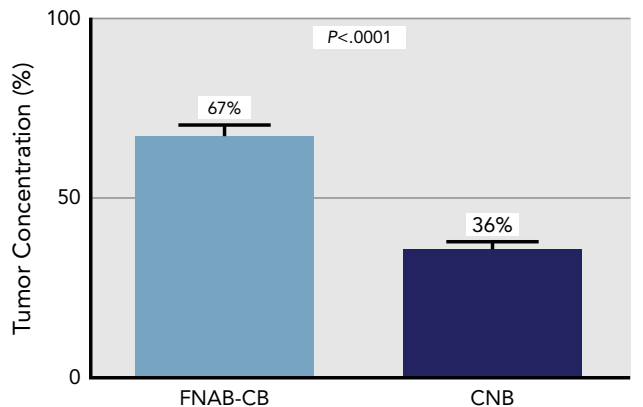

Figure 1. Tumor concentration in FNAB-CB versus CNB specimens. Abbreviations: $\mathrm{CNB}$, core needle biopsy; $\mathrm{FNAB}-\mathrm{CB}$, fine-needle aspiration biopsy with cell block.

exhibited greater tumor cellularity than the CNBs. Thus, after final review including level sections, the FNAB-CBs had superior tumor cellularity in $38 \%$ of cases and the CNBs showed better tumor cellularity in $56 \%$ of cases; equal cellularity was seen in $6 \%$ of cases.

We also analyzed data from 1997 through 2016 for all liver cases that had FNA and CNB performed concurrently, regardless of CBs. At ZSFGH, all cases had available CBs; at UCSFMC, 20 cases lacked CB and diagnosis was based on smears. Of these 20 cases, 6 showed only benign liver on the smears and matched CNBs confirmed these findings; 2 showed lymphoma on smears, in which architecture was deemed important and CNB was obtained in lieu of CB; 1 showed abscess, with material submitted for culture; 5 showed metastatic adenocarcinoma with a known history of breast cancer and the referring clinician requested $\mathrm{CNB}$, and therefore CB was not collected; 2 showed metastatic colon cancer; 1 showed metastatic lung cancer; 1 showed metastatic pancreatic cancer with an established history of cancer and was confirmed by CNB without immunohistochemistry; and 2 showed poorly differentiated carcinoma on both smears and CNB without a known history of cancer and extensive immunohistochemistry was performed on the CNB specimen without a definite classification.

\section{Discussion}

In this era of rapidly evolving personalized medicine, pathologists and interventional radiologists are presented with the challenge of not only providing optimal tissue for traditional microscopic evaluation but also adequate samples for myriad molecular-based testing using minimally invasive and cost-effective biopsy techniques. The 2 most common biopsy methods are $\mathrm{CNB}$ and FNAB. Although both techniques are well established, there is a common misconception that, because of the smaller needle bore, FNAB is not suitable for retrieving adequate amounts of tissue. We reviewed concurrent $\mathrm{CNB}$ and FNAB specimens from tumors in the

\begin{tabular}{|c|c|c|c|}
\hline \multirow[b]{2}{*}{ Diagnosis } & \multirow[b]{2}{*}{ Cases, $\mathbf{n}$} & \multicolumn{2}{|c|}{$\begin{array}{c}\text { Tumor } \\
\text { Concentration (\%) }\end{array}$} \\
\hline & & FNAB-CB & CNB \\
\hline Adenocarcinoma, NOS & 10 & 41 & 25 \\
\hline Adenocarcinoma, pancreaticobiliary & 10 & 69 & 37 \\
\hline Cholangiocarcinoma & 5 & 64 & 36 \\
\hline Hepatic adenoma & 1 & 99 & 20 \\
\hline Hepatocellular carcinoma & 24 & 64 & 41 \\
\hline Metastatic adenocarcinoma, breast & 16 & 83 & 41 \\
\hline Metastatic adenocarcinoma, NOS & 18 & 61 & 29 \\
\hline $\begin{array}{l}\text { Metastatic adenocarcinoma, } \\
\text { gastrointestinal }\end{array}$ & 6 & 94 & 32 \\
\hline $\begin{array}{l}\text { Metastatic high-grade serous } \\
\text { carcinoma }\end{array}$ & 1 & 85 & 20 \\
\hline Metastatic adenocarcinoma, prostate & 1 & 90 & 60 \\
\hline $\begin{array}{l}\text { Metastatic neuroendocrine tumor/ } \\
\text { carcinoma }\end{array}$ & 12 & 74 & 46 \\
\hline Lymphoma & 2 & 23 & 30 \\
\hline Metastatic nasopharyngeal carcinoma & 3 & 60 & 57 \\
\hline Metastatic melanoma & 3 & 65 & 47 \\
\hline Metastatic squamous cell carcinoma & 2 & 55 & 55 \\
\hline Metastatic gastrointestinal tumor & 2 & 83 & 27 \\
\hline Malignant neoplasm, NOS & 5 & 77 & 22 \\
\hline
\end{tabular}

Abbreviations: CNB, core needle biopsy; FNAB-CB, fine-needle aspiration biopsy with cell block; NOS, not otherwise specified.

liver and showed that both modalities provide sufficient material to make accurate, traditional microscopic diagnoses. In addition, we showed that FNAB-CBs have superior tumor concentration compared with concurrent CNBs, and that in at least $40 \%$ of the study cases, FNA-CB tumor cellularity was equal to or greater than that of CNB.

The potential impact of these findings on patient care is significant. Ideally, FNAB specimens can be the sole tissue source for diagnostic testing and all ancillary studies needed for deciding optimal treatment. However, in current practice where both biopsy techniques are often used, FNAB-CB and smears could serve as important resources, particularly for molecular/nucleic acid-based testing. In addition to showing that FNAB-CBs have superior tumor concentration compared with $\mathrm{CNB}$, we also showed that initial processing of FNAB-CB specimens may lead to an underestimation of relative tumor abundance. Therefore, if CNB tissue has been exhausted in the process of applying immunohistochemistry stains to determine tumor type/site of origin and additional tissue is needed for discovering therapeutic targets, then subjecting a patient to another biopsy may not be necessary if an FNAB-CB specimen is available. Similarly, clinical trials 
A

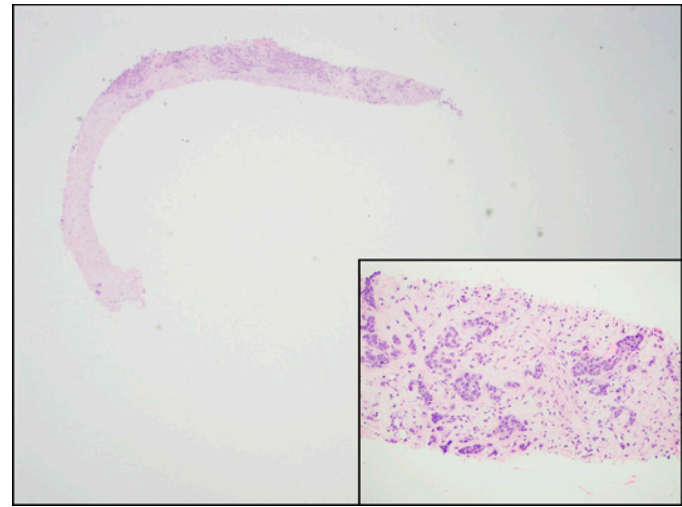

B

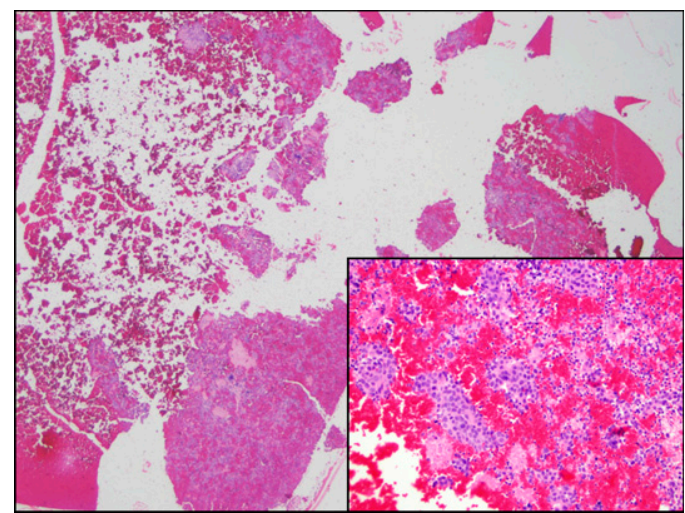

C

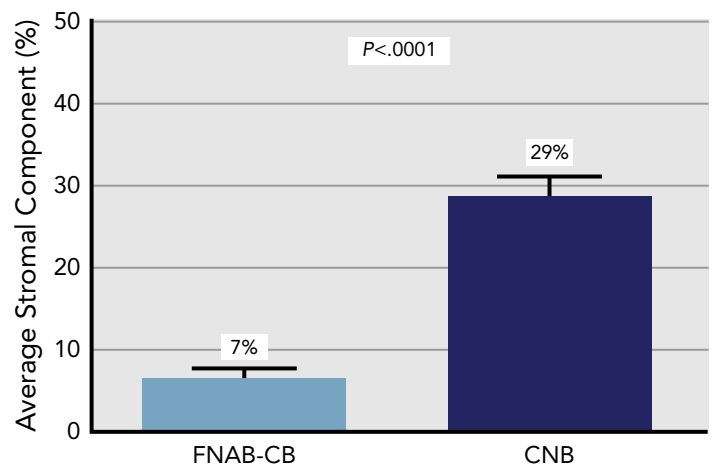

Figure 2. Stromal component of $F N A B-C B$ versus $C N B$. (A) $C N B$ and (B) FNAB-CB of metastatic breast adenocarcinoma (both $A$ and $B$ : hematoxylin-eosin, original magnification $\times 2$; inset magnification $\times 20$ ). (C) Average stromal component in FNAB-CB versus CNB.

Abbreviations: $C N B$, core needle biopsy; FNAB-CB, fine-needle aspiration biopsy with cell block.

could use available FNAB specimens to enroll patients instead of considering a rebiopsy, which not only delays treatment but also adds cost and subjects the patient to unnecessary risk for procedural complications, including bleeding, pain, and pneumothorax. ${ }^{15,16}$ In comparing FNAB-CB and CNB whole sections, our study
A

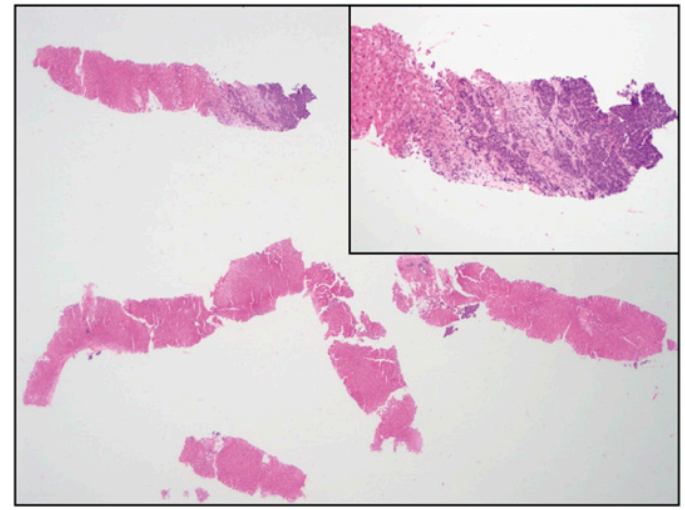

B

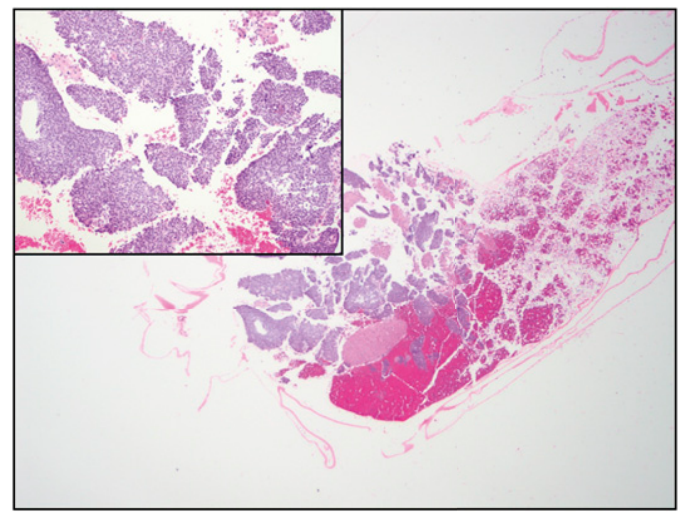

C

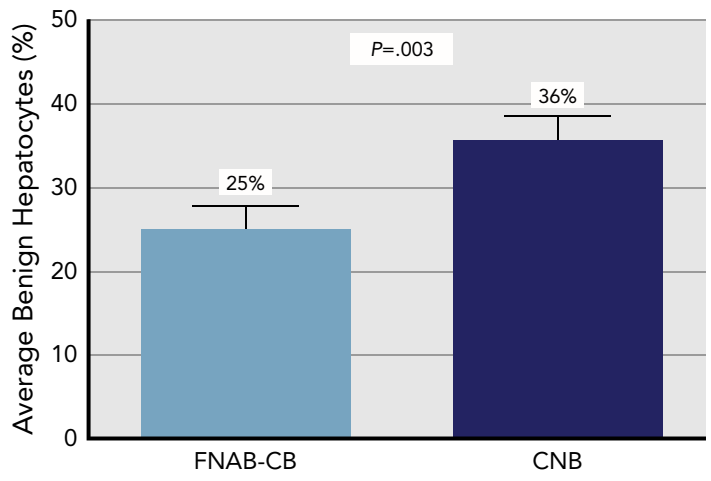

Figure 3. Hepatocyte component of FNAB-CB versus $C N B$. (A) $C N B$ and (B) FNAB-CB of metastatic adenocarcinoma, NOS (both A and B: hematoxylin-eosin, original magnification $\times 2$; inset $\times 10$ ).

(C) Average benign hepatocyte component in FNAB-CB versus CNB Abbreviations: CNB, core needle biopsy; FNAB-CB, fine-needle aspiration biopsy with cell block; NOS, not otherwise specified.

showed that $43 \%$ of samples from CNBs and $25 \%$ from FNAB-CBs did not meet the common $20 \%$ tumor cell fraction cutoff for molecular testing. Microdissection of the blocks/sections, which was not attempted in this study, has the potential to improve these numbers for both types of specimens. ${ }^{17}$ Other studies ${ }^{10,11}$ have shown 
that smeared tumor cells from FNAB are well suited to molecular studies and can further improve chances for successful testing.

The ability to render a diagnosis based on morphologic findings alone is no longer the norm. Immunohistochemistry stains, fluorescence in situ hybridization, and NGS and similar gene-based testing are becoming increasingly common because of diagnostic, prognostic, and therapeutic implications. Although CNBs are commonly preferred for these ancillary tests, tumor cell quantity and concentration in particular can be suboptimal, especially in stroma-rich tumors. Because FNAB-CBs are also FFPE, tests validated for CNBs can also be performed on FNAB-CBs. Therefore, the recommendation to test lung cancers for therapeutic targets has been changed from recommending CNB exclusively to also including FNABs. ${ }^{18}$ Additionally, changes are occurring in clinical trial settings. In 2016, an interim analysis of the phase II NCI-MATCH trial showed that by adding FNAB material including smears to $\mathrm{CNB}$, the percentage of cases with material allowing complete tumor testing increased from $87 \%$ to $99 \% .^{19}$

NGS is a valuable tool that is revolutionizing medicine and becoming part of routine clinical care. Although NGS platforms vary in their analytical sensitivity, one of the goals is their ability to detect low-frequency variants and avoid false-negative results. In general, the reported tumor fraction necessary to achieve NGS success is $>20 \% .^{12,20,21}$ Our study shows an average tumor fraction of $67 \%$ in FNAB-CBs (vs $36 \%$ in CNBs). Furthermore, $43 \%(n=59)$ of the CNB specimens exhibited tumor fractions $\leq 20 \%$ compared with $25 \%(n=34)$ of FNAB-CBs, which suggests that a large number of CNBs would not meet adequacy standards for NGS. Given that CNBs and FNAB-CBs are both FFPE samples, one would assume that quality metrics would also be similar when an adequate amount and concentration of tumor cells are present. This was confirmed in several recent studies that showed no significant difference in initial DNA concentration or NGS success in FNAB-CBs compared with CNBs. ${ }^{11,22-24}$

FNAB sample quality is determined by several factors, including proficiency in sampling technique and sample preparation, use of ROSE in real time to verify correct needle placement and sample adequacy, and optimal specimen triaging, including dedicated passes for CB material and other ancillary tests based on morphologic features evaluated as part of ROSE. At our hospitals, deep-seated FNABs are performed by interventional radiologists, with cytopathology staff present to prepare samples. Cytopathologists review samples in the radiology suite or remotely via imaging transfer, allowing real-time feedback on sample adequacy. Thus, our results reflect collaborative efforts between skilled radiologists and cytopathologists. We acknowledge, however, that some practices may not have cytopathologists available for ROSE and may default to performing CNBs instead.

\section{Conclusions}

This study compared concurrently obtained CNBs and FNAB-CBs in terms of diagnostic accuracy, tumor cell concentration, and overall tumor cellularity. Although both biopsy techniques are adequate for diagnosis, we showed that FNAB-CB provided superior tumor cell concentration and overall cellularity in a large subset, thus making it an excellent substrate for NGS and similar studies. FNAB should be strongly considered as a sampling modality, especially in patients who are candidates for molecular testing that requires at least $20 \%$ tumor cells in the specimen.

Our results, coupled with the preferred safety profile of FNABs, underscore the value of cytopathology in current practice. Although FNAB specimens are currently underused, they have the potential to play an important role in facilitating testing needed for personalized medicine, and thus significantly contributing to optimal patient care. The radiologist performing a percutaneous biopsy is often the one to determine whether FNAB, CNB, or both are performed. This report presents data underscoring the value of FNAB with $\mathrm{CB}$ preparation in the era of personalized medicine.

Submitted July 6, 2018; accepted for publication March 25, 2019.

Author contributions: Study concept: Ljung. Study design: All authors. Data acquisition and entry: Goldhoff, Vohra. Data analysis: Goldhoff, Ljung. Radiology review: Kolli. Drafting of manuscript: Goldhoff. Manuscript review and editing: All authors.

Disclosures: The authors have disclosed that they have not received any financial consideration from any person or organization to support the preparation, analysis, results, or discussion of this article.

Correspondence: Britt-Marie Ljung, MD, Department of Pathology, Mission Bay Campus, University of California San Francisco, 1825 4th Street, Room L 2181C, Box 1785, San Francisco, CA 94143. Email: Britt-Marie.Ljung@ucsf.edu

\section{References}

1. Kwan SW, Bhargavan M, Kerlan RK Jr, et al. Effect of advanced imaging technology on how biopsies are done and who does them. Radiology 2010;256:751-758.

2. Hahn PF, Guimaraes AR, Arellano RS, et al. Nonvascular interventional procedures in an urban general hospital: analysis of 2001-2010

with comparison to the previous decade. Acad Radiol 2015;22 904-908.

3. Marshall D, Laberge JM, Firetag B, et al. The changing face of percutaneous image-guided biopsy: molecular profiling and genomic analysis in current practice. J Vasc Interv Radiol 2013;24:1094-1103. 
4. Liye S, Chang R, Padmanabhan V, et al. For diagnosis of liver masses, fineneedle aspiration versus needle core biopsy: which is better? J Am Soc Cytopathol 2018;7:46-49.

5. Florentine BD, Staymates B, Rabadi M, et al. The reliability of fine-needle aspiration biopsy as the initial diagnostic procedure for palpable masses: a 4-year experience of 730 patients from a community hospital-based outpatient aspiration biopsy clinic. Cancer 2006;107:406-416.

6. Masood S, Rosa M, Kraemer DF, et al. Comparative cost-effectiveness of fine needle aspiration biopsy versus image-guided biopsy, and open surgical biopsy in the evaluation of breast cancer in the era of Affordable Care Act: a changing landscape. Diagn Cytopathol 2015;43:605-612.

7. Capalbo E, Peli M, Lovisatti M, et al. Trans-thoracic biopsy of lung lesions: FNAB or CNB? Our experience and review of the literature. Radiol Med (Torino) 2014;119:572-594

8. Coley SM, Crapanzano JP, Saqi A. FNA, core biopsy, or both for the diagnosis of lung carcinoma: obtaining sufficient tissue for a specific diagnosis and molecular testing. Cancer Cytopathol 2015;123:318-326.

9. Brachtel EF, Operaña TN, Sullivan PS, et al. Molecular classification of cancer with the 92-gene assay in cytology and limited tissue samples. Oncotarget 2016;7:27220-27231.

10. Billah S, Stewart J, Staerkel G, et al. EGFR and KRAS mutations in lung carcinoma: molecular testing by using cytology specimens. Cancer Cytopathol 2011;119:111-117.

11. Roy-Chowdhuri S, Chen H, Singh RR, et al. Concurrent fine needle aspirations and core needle biopsies: a comparative study of substrates for next-generation sequencing in solid organ malignancies. Mod Pathol 2017;30:499-508.

12. Roy-Chowdhuri S, Stewart J. Preanalytic variables in cytology: lessons learned from next-generation sequencing - the MD Anderson experience. Arch Pathol Lab Med 2016;140:1191-1199.

13. Bellevicine C, Malapelle U, Vigliar E, et al. How to prepare cytological samples for molecular testing. J Clin Pathol 2017;70:819-826.

14. Balassanian R, Wool GD, Ono JC, et al. A superior method for cell block preparation for fine-needle aspiration biopsies. Cancer Cytopathol 2016; 124:508-518.
15. van Zante A, Ljung BM. Fine-needle aspiration versus core needle biopsy: reconsidering the evidence of superiority. Cancer Cytopathol 2016;124 853-856.

16. Heerink WJ, de Bock GH, de Jonge GJ, et al. Complication rates of CT-guided transthoracic lung biopsy: meta-analysis. Eur Radiol 2017;27: 138-148.

17. Domazet B, MacLennan GT, Lopez-Beltran A, et al. Laser capture microdissection in the genomic and proteomic era: targeting the genetic basis of cancer. Int J Clin Exp Pathol 2008;1:475-488.

18. Lindeman NI, Cagle PT, Beasley MB, et al. Molecular testing guideline for selection of lung cancer patients for EGFR and ALK tyrosine kinase inhibitors: guideline from the College of American Pathologists, International Association for the Study of Lung Cancer, and Association for Molecular Pathology. J Thorac Oncol 2013;8:823-859.

19. Flaherty KT, Chen AP, O'Dwyer PJ, et al. NCl-Molecular Analysis for Therapy Choice (NCI-MATCH or EAY131): interim analysis results. Presented at the 107th Annual Meeting of American Association for Cance Research; April 16-20, 2016; New Orleans, Louisiana.

20. Chen H, Luthra R, Goswami RS, et al. Analysis of pre-analytic factors affecting the success of clinical next-generation sequencing of solid organ malignancies. Cancers (Basel) 2015;7:1699-1715.

21. Goswami RS, Luthra R, Singh RR, et al. Identification of factors affecting the success of next-generation sequencing testing in solid tumors. Am J Clin Pathol 2016;145:222-237.

22. Hwang DH, Garcia EP, Ducar MD, et al. Next-generation sequencing of cytologic preparations: an analysis of quality metrics. Cancer Cytopatho 2017;125:786-794.

23. Gailey MP, Stence AA, Jensen CS, et al. Multiplatform comparison of molecular oncology tests performed on cytology specimens and formalinfixed, paraffin-embedded tissue. Cancer Cytopathol 2015;123:30-39.

24. Shamanna RK, Portier BP, Singh RR, et al. Next-generation sequencingbased multi-gene mutation profiling of solid tumors using fine needle aspiration samples: promises and challenges for routine clinical diagnostics. Mod Pathol 2014;27:314-327.

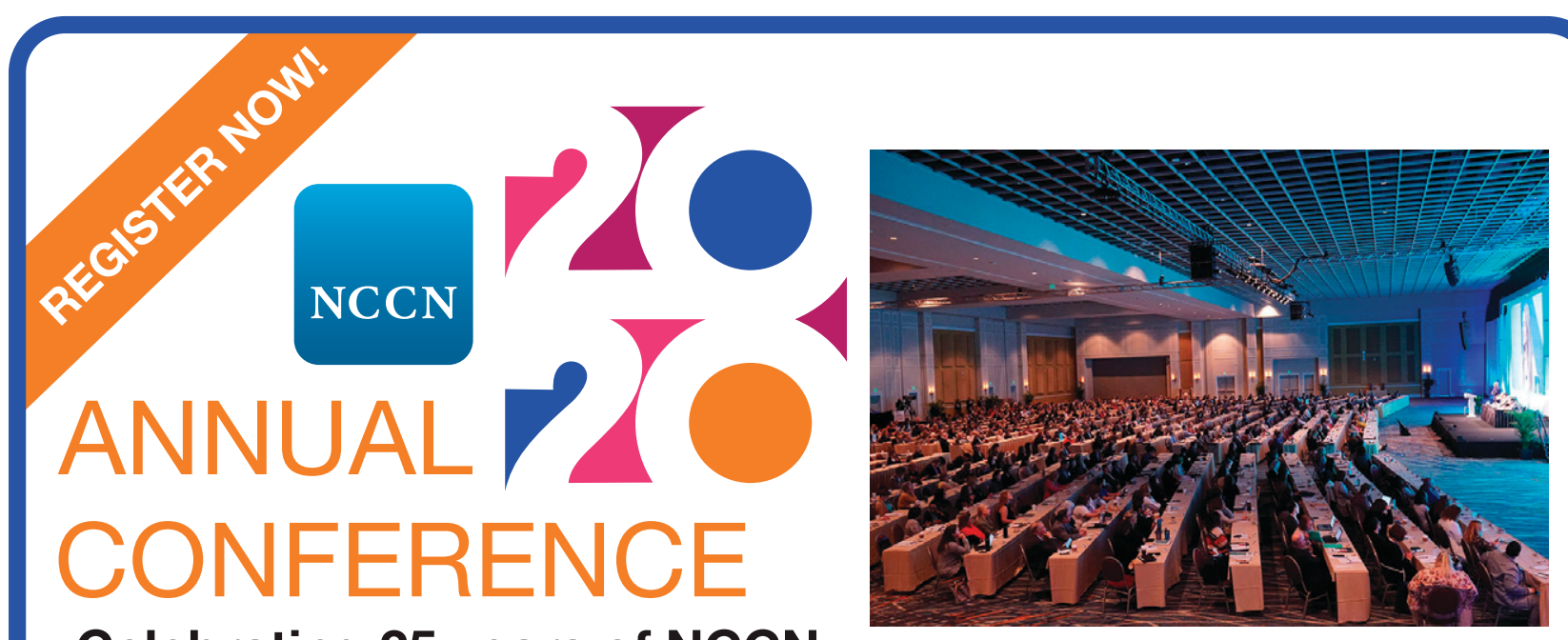

-Celebrating 25 years of NCCN.

March 20 - 22, $2020 \bullet$ Rosen Shingle Creek • Orlando, FL

\section{New Schedule • New Offerings • More Options NCCN.org/conference}

